\title{
Picosecond, ultraviolet fiber laser at 300MHz repetition rate: resonant quantum logic gate source.
}

\author{
Mahmood Irtiza Hussain ${ }^{1}$, Matthew Joseph Petrasiunas ${ }^{1}$, Mirko Lobino ${ }^{1}$, Erik \\ Streed $^{1,2}$ and David Kielpinski ${ }^{1}$ \\ ${ }^{1}$ Center for Quantum Dynamics, Griffith University, Nathan, QLD 4111, Australia \\ ${ }^{2}$ Institute for Glycomics, Griffith University, Gold Coast, QLD 4222 Australia \\ *mahmood.hussain2@griffithuni.edu.au
}

\begin{abstract}
We engineered a fiber laser source capable to produce $2.5 \mathrm{ps}$ UV pulses at $300 \mathrm{MHz}$ repetition rate. Laser wavelength resonates with one of the strong transition in $\mathrm{Yb}^{+}$ion, and it will enable us to coherently manipulate $\mathrm{Yb}^{+}$via $\pi$-transitions to make fast entangling gates.
\end{abstract}

OCIS codes: (320.7090), (260.7190), (190.4370), (190.7220), (270.5585).

\section{Introduction}

Trapped-ions are one of the major candidates for quantum computing. Successful implementation of such quantum systems require simultaneous presence of entanglement and scalability, which is hard due to enhanced decoherence. There are experimental studies, which demonstrates ultrafast gates for single ion [1]. Record time for two qubit entanglement is about $35 \mu \mathrm{s}$, [2] which is long to realize useful quantum computation. Nonetheless, an idea was proposed to speed-up the entangling gate time [3], gist of the proposal is to shine counter-propagating ultrafast and resonant laser pulses (interspersed in time with some sequences) with trapped-ions. Such lasers are not readily available due to limited wavelength selection, repetition rate and power level. Here, we proposed a fully scalable fiber laser source which can generate $2.5 \mathrm{ps}, 300 \mathrm{MHz}$ repetition rate pulses at $369.53 \mathrm{~nm}$ wavelength. We specifically shape laser wavelength to $369.53 \mathrm{~nm}$, because it resonates with $\mathrm{S}_{1 / 2}-\mathrm{P}_{1 / 2}$ energy levels in $\mathrm{Yb}^{+}$ion. Laser source has been integrated with trapped-ion setup and very soon going to demonstrate a single pulse as a $\pi$-pulse for coherent manipulation of $\mathrm{Yb}^{+}$. It is possible to generate $\pi$-pulse with pulse energy $<0.1 \mathrm{~nJ}$, however laser source can produce pulse energy $>0.5 \mathrm{~nJ}$. Hence, it shows the viability of the laser system to establish $\pi$-transitions. After this demonstration we will investigate resonant fast entangling gates.

\section{Experimental Work}

Laser starts with an Er-doped linear fiber laser operating at $1564 \mathrm{~nm}$, which is a passively mode-locked, pumped by $976 \mathrm{~nm}$ diode, as shown in Fig 1a. Fiber lengths are carefully chosen to balance dispersion and non-linearity. By altering pump current and birefringence we produced harmonics and increased the repetition rate to $300 \mathrm{MHz}$. Repetition rate was locked by introducing a piezo mounted mirror (PMM) and Proportional-integral-differential (PID) controller as shown in the Fig. 1c. Pulses out of seed laser are used to generate octave span supercontinuum (1000nm $<$ $\lambda<2000 \mathrm{~nm}$ ) and 1108.6nm light is separated by spectral slicing. Later on pulses were amplified and compressed for second harmonic generation (SHG) at 554.3nm [4]. We split up the pump and second harmonic beams after SHG using dichroic mirror and steer them to propagate in separate beam paths. Later on, we mixed them via sum frequency generation (SFG) to generate UV pulses as shown in the Fig. 1b. We used critical phase matched $\mathrm{LiB}_{3} \mathrm{O}_{5}(\mathrm{LBO})$ crystal. Beams after exiting PPSLT crystal are focused to spot sizes (second harmonic $=35 \mu \mathrm{m}$, residual $\mathrm{IR}=40 \mu \mathrm{m}$ ) @ $1 / \mathrm{e}^{2}$ radius inside $\mathrm{LBO}$, where each beam carries maximum peak power of $(1.74 \mathrm{KW}, 2.9 \mathrm{KW})$, corresponds to peak intensities of $\left(35 \mathrm{MW} / \mathrm{cm}^{2}, 57 \mathrm{MW} / \mathrm{cm}^{2}\right)$. SFG-pump beams are temporally and spatially overlapped to produce maximum of $190 \mathrm{~mW} \mathrm{UV} \mathrm{and} \mathrm{it} \mathrm{relates} \mathrm{to} \mathrm{single} \mathrm{pass} \mathrm{UV} \mathrm{conversion} \mathrm{efficiency}\left(\eta=P_{370 \mathrm{~nm}} /\left(P_{1108 \mathrm{~nm}}+P_{554 \mathrm{~nm}}\right)\right)$ of $6 \%$ in SFG. Immediately after UV filtration, telescope consists of cylindrical and plano-convex lenses is placed in the beam path for beam profile correction and collimation as shown in Fig 1 b. Poor beam quality $\left(\mathrm{M}^{2}>2\right)$ clearly indicates that spatial walk-off in the pump beams impair beam overlap and hence conversion efficiency. After beam shaper $\mathrm{M}^{2}$ was improved to 1.5 , still bit high, however it will not have significant effect in $\pi$-pulse accomplishment. 


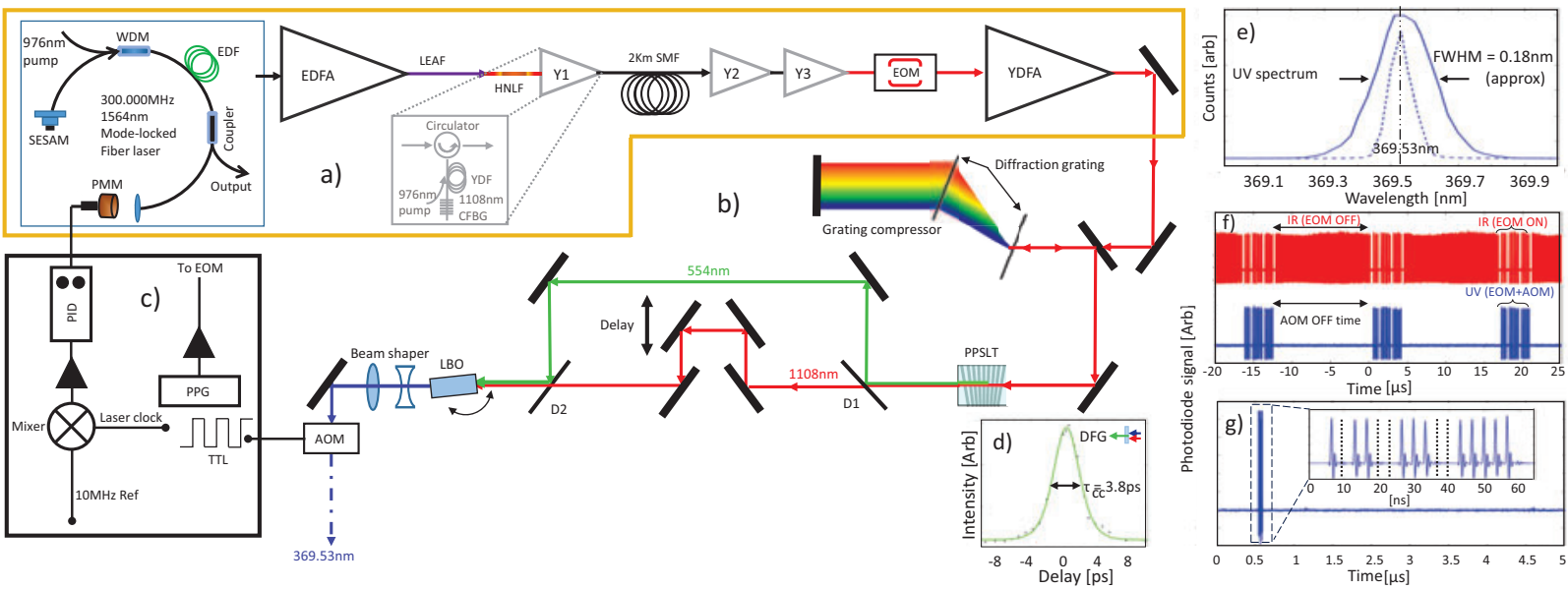

Fig. 1. (a),(b) and (c) Schematic of laser system. LEAF: Large effective area fiber, WDM: Wavelength division multiplexer, SESAM: Semiconductor saturable absorber mirror. (d) CC envelope showing CC duration of 3.8ps, corresponds to actual duration of $2.5 \mathrm{ps}$ (e) UV spectrum speak roughly same as peak of UV laser locked with $\mathrm{Yb}^{+} 369.53 \mathrm{~nm}$ transition. (f) and (g) Switched UV pulses with arbitrary pattern. Black dot lines in inset shows OFF UV pulses.

UV center wavelength can be tuned to exact resonance by slight variation in the crystal phase matching (within acceptance bandwidth) or by heating/cooling the CFBG. When laser is fully optimized, UV power-stability remains within $5 \%$ of maximum average power over many hours. UV pulse duration has been estimated by cross-correlation (CC) technique. Unconverted IR and UV pulses after SFG are separated and used for difference frequency generation (DFG) at 554nm, which from CC envelope with delay in IR pulses as shown in the Fig. 1d. Since actual CC duration and IR pulse duration are roughly same which gives us a straight forward estimate of UV duration to be $\approx 2.5 \mathrm{ps}$. DFG was generated by $\mathrm{LiIO}_{3}$ crystal.

Fast UV pulse-switching was done by the electro-optic modulators(EOM) and acousto-optic modulators(AOM). EOM produces fast switching signals driven by the amplified RF output of the $12.5 \mathrm{GHz}$ programmable patten generator (PPG), while TTL signals were feeded to AOM. After syncing AOM and EOM switching time we produced many and few UV pulse-switching patterns as shown in the Fig. 1(f,g).

\section{Conclusion}

In summary, we developed fully scalable fiber laser source which can generate $369.53 \mathrm{~nm}, 2.5 \mathrm{ps}$ pulses at $300 \mathrm{MHz}$ repetition rate. Laser system could have many potential applications e.g., frequency tripling of 1905nm wavelength could be a novel source for Hydrogen cooling and multiple wavelength channels can run applications in parallel. Here, we shape the laser system to resonate with strong $\mathrm{Yb}^{+}$transition, which can serve as a platform to make resonant quantum logic gates, atom interferometry and single atom auto-correlator.

\section{References}

1. W. C. Campbell, J. Mizrahi, Q. Quraishi, C. Senko, D. Hayes, D. Hucul, D. N. Matsukevich, P. Maunz, and C. Monroe, “Ultrafast gates for single atomic qubits," Phys. Rev. Lett. 105, 090502 (2010).

2. D. Leibfried, B. DeMarco, V. Meyer, D. Lucas, M. Barrett, J. Britton, W. M. Itano, B. Jelenković, C. Langer, T. Rosenband, and D. J. Wineland, "Experimental demonstration of a robust, high-fidelity geometric two ion-qubit phase gate," Nature 422, 412-415 (2003).

3. J. J. Garcia-Ripoll, P. Zoller, and J. I. Cirac, "Speed optimized two-qubit gates with laser coherent control techniques for ion trap quantum computing,” Phys. Rev. Lett. 91, 157901 (2003).

4. M. J. Petrasiunas, M. I. Hussain, J. Canning, M. Stevenson, and D. Kielpinski, "Picosecond 554nm yellow-green fiber laser source with average power over 1 W," Opt. Express 22, 17716-17722 (2014). 